\title{
THE INFLUENCE OF FREQUENCY ON THE RESISTANCE AND INDUCTANCE OF SOLENOIDAL COILS.
}

\author{
By Louis Cohen.
}

The resistance and inductance of a conductor depend on the distribution of the current within the conductor. When the current is continuous the distribution is uniform throughout the section of the conductor, and the problem of finding the resistance and inductance is a very simple one. If, however, the current is varying with respect to time, eddy currents with their accompanying magnetic fields will be generated within the conductor, which will alter the distribution of the current and consequently change the resistance and inductance of the conductor. The problem of finding the law of variation of resistance and inductance with the frequency is of considerable importance, and for the case of a straight cylindrical wire the problem has been satisfactorily worked out. The results, however, as obtained for the case of a straight wire, will not hold when the same wire is wound into a coil. It has been found experimentally that the change in resistance of a coil for any given frequency is much larger than if the winding of the coil were drawn out into a straight conductor. Dolezalek, ${ }^{1}$ in his experiments with high-frequency currents, was the first to call attention to the fact that the change of resistance is larger than that calulated by the theory for straight conductors. Battelli and Maggi have also carried out a series of experiments along that line and found the same results.

Owing to the importance of the problem in its application to wireless telegraphy, several investigators have sought to obtain solutions to this problem. Their efforts were not attended by any

\footnotetext{
${ }^{1}$ Dolezalek, Annalen der Physik, 12; I903.
} 
high degree of success, inasmuch as the results obtained do not agree with experimental facts.

Max Wien, ${ }^{2}$ who first investigated the problem, obtained a solution in a form which is rather a slow convergent series, and the calculation of the various terms is very laborious. Independently of this inconvenience, which Wien himself pointed out, his theory is not correct, as was shown by Battelli, since he implicitly assumes that the distribution of the current in the section of the wire is the same at all points at the same distance from the axis. Sommerfeld, ${ }^{3}$ who made the next attempt to get a solution to the problem, also failed to obtain an agreement between his theoretical conclusions and experiment. According to Sommerfeld's theory, the change of resistance of a coil will be independent of the width of the winding - that is, if we replace one turn of ro $\mathrm{mm}$ in width by ten turns of I $\mathrm{mm}$ in width, but of the same thickness, then the change in resistance will, in accordance with Sommerfeld's theory, be the same. This appears to the anthor to be incorrect; for apriori we can say that the change of resistance must be a function of the section of the winding. Recently another solution of the problem was given by G. Picciati, ${ }^{4}$ but unfortunately his theoretical results fail also to agree with experimental results.

The table on page $\mathrm{r} 7 \mathrm{O}$ shows to what extent the values calculated by the various formulas, for the per cent change in resistance, differs from the experimental values. From the table it can readily be seen that none of the formulas derived by the various investigators agree with experimental results.

The question of change of self-inductance is not of such practical importance as the change in resistance, and there are no experimental results on record by which we could verify any theory. The problem was discussed by Wien, but the same criticism which was offered to his work on the change of resistance will also apply to his work on the change of inductance. Doctor Coffin ${ }^{5}$ has recently contibuted two papers on the change of inductance of coils with frequency. He has not, however, developed any new theory,

${ }^{2}$ M. Wien, Annalen der Physik, 14, p. I; I904.

${ }^{3}$ Sommerfeld, Annalen der Physik, 15, p. 673; 1904.

${ }^{4}$ G. Picciati, Il Nuovo Cimento (5), 2, p. 35 I.

${ }^{5}$ This Bulletin, 2, p. 275; Physical Review, 22, p. 365 . 
reproducing Sommerfeld's work with such modifications as to obtain the change in inductance in place of resistance.

In view of the above considerations, it appeared to the author desirable to investigate the problem anew, and it was very gratifying to find that the results obtained agree with experiment. $\mathrm{My}$ conclusions are that the change in resistance of a coil is a function of the frequency, the section of the winding, and the pitch of the winding.

Suppose we have a very long solenoid, and the windings very close together, so that we may assume the magnetic field within the solenoid to be uniform all the way through, even close to the winding, and parallel to the axis of $Z$; the field outside the solenoid will be zero. Let us suppose the section of the winding to be square, and consider now the electric and magnetic distribution in a single

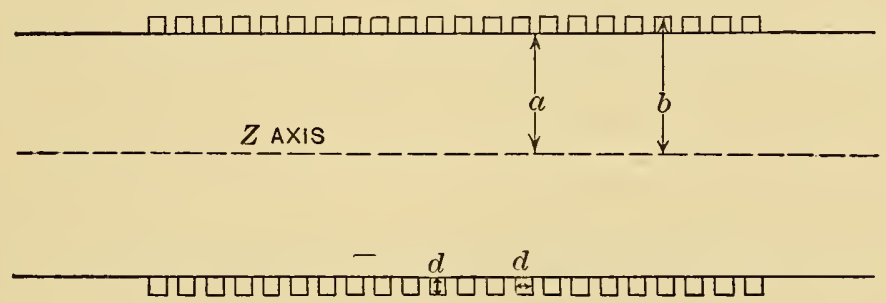

Fig. 1.

winding. The electric force will be circular, centered on the axis of $Z$, while the magnetic force will have two components, one radial and the other longitudinal, parallel to the axis of $Z$.

Let $E$ denote the electric force.

$M$ " the longitudinal component of the magnetic force.

$L$ " the radial component of the magnetic force.

$I_{1}$ " the current at any point in conductor due to variation of magnetic field within conductor.

$I$ " the total current in conductor.

$\sigma$ " $\quad$ the conductivity.

$d$ " width or breadth of section of winding.

The connection between electric and magnetic forces are as follows:

Curl (magnetic force $)=4 \pi \times$ current.

Curl (electric force) $=$ rate of variation of magnetic force with respect to time. 
In this particular case these relations can be expressed in the following form:

$$
\left.\begin{array}{l}
\frac{d M}{d r}-\frac{d L}{d z}=4 \pi \sigma E \\
-\frac{d E}{d z}=\frac{d L}{d t} \\
\frac{d E}{d r}+\frac{E}{r}=\frac{d M}{d t}
\end{array}\right\}
$$

From the above three equations we can easily deduce the following equation of propagation:

$$
\frac{d^{2} E}{d r^{2}}+\frac{\mathrm{I}}{r} \frac{d E}{d r}-\frac{E}{r^{2}}+\frac{d^{2} E}{d z^{2}}=4 \pi \sigma E
$$

If the impressed force is simple harmonic with respect to the time, we can put

$$
E=-u \cos m z e^{i \not p t}
$$

where $u$ is function of $r$ only, and $m$ is any arbitrary constant. Introducing the value of $E$ into equation (2) we get the following:

$$
\begin{gathered}
\frac{d^{2} e}{d r^{2}}+\frac{\mathrm{I}}{r} \frac{d u}{d r}+\left(-k^{2}-\frac{\mathrm{I}}{r^{2}}\right) u=0 \\
k^{2}=m^{2}+4 \pi \sigma i p
\end{gathered}
$$

The solution of equation (3) will be

$$
u=A J_{1}(i k r)+B Y_{1}(i k r)
$$

$J_{1}$ and $Y_{1}$ are the Bessel functions of the first and second kind, and of the first order. The complete solution of equation (2) will be

$$
E=\left\{A J_{1}(i k r)+B Y_{1}(i k r)\right\} \cos m z e^{i p t}
$$

The values of $L$ and $M$ may be obtained from equation (4) by the aid of the relations between the electric and magnetic forces as given by equations ( $\mathrm{I}$ ), thus: 


$$
L=\frac{m}{i p}\left\{A J_{1}(i k r)+B Y_{1}(i k r)\right\} \sin m z e^{i p t}
$$

$$
\begin{gathered}
\frac{d M}{d r}=\frac{d L}{d z}+4 \pi \sigma E=\left(\frac{m^{2}}{i p}+4 \pi \sigma\right)\left\{A J_{1}(i k r)+B Y_{1}(i k r)\right\} \cos m z e^{i p t} . \\
=\frac{k^{2}}{i p}\left\{A J_{1}(i k r)+B Y_{1}(i k r)\right\} \cos m z e^{i p t} \\
M=\frac{k}{p}\left\{A J_{0}(i k r)+B Y_{0}(i k r)\right\} \cos m z e^{i p t}
\end{gathered}
$$

$J_{0}$ and $Y_{0}$ are the Bessel functions of the zero order.

Since $m$ is an arbitrary constant, we can choose the value of $m$ so as to suit the conditions of the problem.

Let us put

$$
m=\frac{n \pi}{d} \quad(n=\mathrm{I}, 3,5,7, \ldots)
$$

and since $k$ is a function of $m, k$ will also have an infinite number of values. If we now put

$$
A=\frac{4}{n \pi}(i)^{n-1} A^{\prime}, B=\frac{4}{n \pi}(i)^{n-1} B^{\prime}
$$

then the values of $E, L$, and $M$ will be given by the following expressions:

$$
\left.\begin{array}{l}
E=\sum_{n=1}^{n=\infty}\left\{A^{\prime}{ }_{n} J_{1}\left(i k_{n} r\right)+B^{\prime}{ }_{n} Y_{1}\left(i k_{n} r\right)\right\} \frac{4}{n \pi}(i)^{n-1} \cos \frac{n \pi z}{d} e^{i p t} \\
L=\sum_{n=1}^{n=\infty} \frac{m_{n}}{i p}\left\{A^{\prime}{ }_{n} J_{1}\left(i k_{n} r\right)+B^{\prime}{ }_{1} Y_{1}\left(i k_{n} r\right)\right\} \frac{4}{n \pi}(i)^{n-1} \sin \frac{n \pi z}{d} e^{i p t} \\
M=\sum_{n=1}^{n=\infty} \frac{k_{n}}{i p}\left\{A^{\prime}{ }_{n} J_{0}\left(i k_{n} r\right)+B^{\prime}{ }_{n} Y_{0}\left(i k_{n} r\right)\right\} \frac{4}{n \pi}(i)^{n-1} \cos \frac{n \pi z}{d} e^{i p t}
\end{array}\right\}
$$

If the solenoid is of considerable length the magnetic field within the solenoid will be constant and equal to the magnetizing force, which is $4 \pi S I$ where $I$ is the total current and $S$ the number of turns per unit length, and the field outside the solenoid will be zero.

At the surfaces of the solenoid, $M$, the longitudinal component of the magnetic force within winding, will be the only one acting; 
and to satisfy the condition of continuity we must have the following relations satisfied:

$$
\left.\begin{array}{l}
\left.\Sigma \frac{k_{n}}{p}\left\{A^{\prime}{ }_{n} J_{0}\left(i k_{n} a\right)+B^{\prime}{ }_{n} Y_{0}\left(i k_{n} a\right)\right\} \frac{4}{n \pi}(i)^{n-1} \cos \frac{n \pi z}{d} e^{i p t}=4 \pi s I e^{i p t}\right\} \\
\Sigma \frac{k_{n}}{p}\left\{A^{\prime}{ }_{n} J_{0}\left(i k_{n} b\right)+B^{\prime}{ }_{n} Y_{0}\left(i k_{n} b\right)\right\} \frac{4}{n \pi}(i)^{n-1} \cos \frac{n \pi z}{d} e^{i p t}=0
\end{array}\right\}
$$

( $a$ is the internal and $b$ the external radius of the solenoid)

These two equations will be satisfied if we determine every set of $A^{\prime}$ and $B^{\prime}$ from the following equations:

$$
\left.\begin{array}{c}
k_{n} p\left\{A^{\prime}{ }_{n} J_{0}\left(i k_{n} a\right)+B^{\prime}{ }_{n} Y_{0}\left(i k_{n} a\right)\right\}=4 \pi s I \\
\left\{A^{\prime}{ }_{n} J_{0}\left(i k_{n} b\right)+B^{\prime}{ }_{n} Y_{0}\left(i k_{n} b\right)\right\}=0
\end{array}\right\}
$$

Then the first equation of (8) will reduce to

$$
4 \pi s I \frac{4}{\pi}\left\{\cos \pi z-\frac{I}{3} \cos \frac{3 \pi z}{d}+\frac{I}{5} \cos \frac{5 \pi z}{d}-\ldots\right\}=4 \pi s I
$$

Now since the series in the brackets for the interval $-\frac{d}{2}$ to $+\frac{d}{2}$ is $\frac{\pi}{4}$, equation (IO) is an identity, and consequently the conditions as expressed by equation (8) will be satisfied.

The constants $A^{\prime}$ and $B^{\prime}$, as determined by equation (9), are as follows:

$$
\left.\begin{array}{rl}
A_{n}^{\prime}= & \frac{\frac{4 \pi s I p}{k_{n}} Y_{0}\left(i k_{n} b\right)}{J_{0}\left(i k_{n} a\right) Y_{0}\left(i k_{n} b\right)-J_{0}\left(i k_{n} b\right) Y_{0}\left(i k_{n} a\right)} \\
B^{\prime}{ }_{n}= & \frac{4 \pi s I p}{k_{n}} J_{0}\left(i k_{n} b\right) \\
J_{0}\left(i k_{n} a\right) Y_{0}\left(i k_{n} b\right)-J_{0}\left(i k_{n} b\right) Y_{0}\left(i k_{n} a\right)
\end{array}\right\}
$$

If we denote by $I_{1}$ the current at any point in the conductor, then

$$
I_{1}=\sigma E=\sigma \sum_{n=1}^{n=\infty}\left\{A^{\prime}{ }_{n} J_{1}\left(i k_{n} r\right)+B^{\prime}{ }_{n} Y_{1}\left(i k_{n} r\right)\right\} \frac{4}{n \pi}(i)^{n-1} \cos \frac{n \pi z}{d} e^{i p t}
$$

The heating effect produced by the current $I_{1}$ in a given volume will be

$$
\iiint \frac{I_{1}^{2} d v}{\sigma}
$$


and it must be remembered that this heating effect is produced by the eddy currents only. In addition to that there is of course a heating effect due to the current flow in the circuit.

Considering, however, only the eddy currents, we may look upon the heating effect as if we had a resistance $R$ and a current $I$ passing through it that is

$$
\begin{aligned}
& \iiint \frac{I_{1}^{2} d v}{\sigma}=R^{\prime} I^{2} \\
& R^{\prime}=\frac{I}{I^{2}} \iiint \frac{I_{1}^{2} d v}{\sigma}
\end{aligned}
$$

Considering our volume as that of a single turn, we have

$$
R^{\prime}=\int_{0}^{2 \pi} \int_{a}^{b} \int_{-\frac{2}{d}}^{\frac{d}{2}} \frac{I_{1}^{2} r d r d z d \theta}{\sigma}
$$

Introducing the value $I^{2}{ }_{1}$, we get

$$
R^{\prime}=\mathrm{I} 6 \pi^{2} \sigma p^{2} s^{2} \int_{0}^{2 \pi} \int_{a}^{b} \int_{-\frac{d}{2}}^{+\frac{d}{2}} \sum_{n=1}^{n=\infty} \frac{\mathrm{I}}{k_{n}}
$$

$\left.\frac{Y_{0}\left(i k_{n} b\right) J_{1}\left(i k_{n} r\right)-J_{0}\left(i k_{n} b\right) Y_{0}\left(i k_{n} r\right)}{J_{0}\left(i k_{n} a\right) Y_{0}\left(i k_{n} b\right)-J_{0}\left(i k_{n} b\right) Y_{0}\left(i k_{n} a\right)} \frac{4}{n \pi}{ }^{(i)^{u-1}} \cos \frac{n \pi z}{d}\right]^{2} r d r d \theta d z($ I 2$)$

Since, however,

$$
\int_{-\frac{d}{2}}^{+\frac{d}{2}} \cos p \frac{\pi z}{d} \cos q \frac{\pi z}{d} d z=0
$$

Where $p$ and $q$ are two distinct odd numbers, therefore in calculating (I 2) we must only consider the square of each term and neglect the product terms, and therefore

$$
\begin{gathered}
R^{\prime}=256 s^{2} p^{2} \sigma 2 \pi \int_{a}^{b} \int_{-\frac{d}{2}}^{\frac{d}{2}} \sum_{n=1}^{n=\infty} \frac{\mathrm{I}}{n^{2} k_{n}{ }^{2}} \\
\left\{\frac{Y_{0}\left(i k_{n} b\right) J_{0}\left(i k_{n} r\right)-J_{0}\left(i k_{n} b\right) Y_{0}\left(i k_{n} r\right)}{J_{0}\left(i k_{n} a\right) Y_{0}\left(i k_{n} b\right)-J_{0}\left(i k_{n} b\right) Y_{0}\left(i k_{n} a\right)}\right\}^{2}(i)^{2 n-2} \cos ^{2} \frac{n \pi z}{d} r d r d z
\end{gathered}
$$




$$
\begin{gathered}
=256 s^{2} p^{2} \sigma \pi d \int_{a}^{b} \sum_{n=1}^{n=\infty} \frac{\mathrm{I}}{n^{2} k_{n}{ }^{2}} \\
\left\{\frac{Y_{0}\left(i k_{n} b\right) J_{0}\left(i k_{n} r\right)-J_{0}\left(i k_{n} b\right) Y_{0}\left(i k_{n} r\right)}{J_{0}\left(i k_{n} a\right) Y_{0}\left(i k_{n} b\right)-J_{0}\left(i k_{n} b\right) Y_{0}\left(i k_{n} a\right)}\right\}^{2}(i)^{2}{ }^{n-2} r d r
\end{gathered}
$$

Now,

and, therefore,

$$
\begin{aligned}
k^{2}=m^{2}+4 \pi \sigma i p & =(a+i \beta) \text { say } \\
\because a^{2}-\beta^{2} & =m^{2} \\
2 a \beta & =4 \pi \sigma p
\end{aligned}
$$

$$
\begin{aligned}
& a=\sqrt{m^{2}+\sqrt{\frac{m^{4}+\mathrm{I} 6 \pi^{2} \sigma^{2} p^{2}}{2}}} \\
& \beta=\sqrt{-m^{2}+\sqrt{\frac{m^{4}+\mathrm{I} 6 \pi^{2} \sigma^{2} p^{2}}{2}}}
\end{aligned}
$$

Even if we shall neglect $m^{4}$ as compared with $\mathrm{I} 6 \pi^{2} \sigma^{2} p^{2}$, then the real part of $k$ will be

$$
a=\sqrt{2 \pi \sigma p}=\sqrt{4 \pi^{2} \sigma f}
$$

where $f$ is the frequency.

If the winding is of copper, then $\sigma=5.9 \times \mathrm{IO}^{-4}$, approximately, and if we assume $f$ to be 2,000 , then,

$$
a=2 \sqrt{5.9 \times \mathrm{IO}^{--4} \times \mathrm{IO} \times 2 \times \mathrm{IO}^{3}}=7 \text {, approximately. }
$$

If $r=5 \mathrm{~cm}$, say, then the real part of $k r$ will be a large quantity, and we may therefore substitute for the Bessel's functions their approximate values, which are as follows: ${ }^{6}$

$$
\begin{array}{cc}
J_{0}(i k r)=\frac{e^{k r}}{\sqrt{2 \pi k r}}, & J_{1}(i k r)=\frac{i e^{k r}}{\sqrt{2 \pi k r}} \\
Y_{0}(i k r)=\frac{e^{-k r} \sqrt{\pi},}{\sqrt{2 k r}} & Y_{1}(i k r)=\frac{-i e^{-k r} \sqrt{\pi} .}{\sqrt{2 k r}} .
\end{array}
$$

${ }^{6}$ See Gray and Mathews Treatise on Bessel Functions, p. I56. 
Introducing these values in equation ( $\mathrm{I}_{3}$ ) we obtain the following:

$$
\begin{array}{r}
R^{\prime}=256 s^{2} p^{2} \pi d \sigma \int_{a}^{b} \sum_{n=1}^{n=\infty} \frac{\mathrm{I}}{n^{2} k^{2}}\left\{\begin{array}{l}
e^{k_{n}(b-r)}+e^{-k_{n}(b-r)} \\
e^{-k_{n} d}-e^{+k_{n} d}
\end{array}\right\}^{2}(i)^{2 n-2} r \frac{a}{r} d r \\
=256 s^{2} p^{2} \pi d a \sigma \int_{a}^{b-a=d)} \sum_{n=1}^{n=\infty} \frac{\mathrm{I}}{n^{2}\left(a_{n}+i \beta_{n}\right)^{2}} \\
\cdot\left\{\frac{e^{\left(a_{n}+i \beta_{n}\right)(b-r)}+e^{-\left(a_{n}+i \beta_{n}\right)(b-r)}}{e^{-\left(a_{n}+i \beta_{n}\right) d}-e^{\left(a_{n}+i \beta_{n}\right) d}}\right\}^{2} d r
\end{array}
$$

In working, however, with complex quantities the real part of the squares of a complex quantity can be obtained by multiplying the quantity by its conjugate. In this case the conjugate is obtained by substituting $-i$ for $i$ everywhere in the expression, and this will give

$$
\begin{aligned}
& R^{\prime}=256 s^{2} p^{2} \pi d a \sigma \int_{a}^{b} \sum_{n=1}^{n=\infty} \frac{\mathrm{I}}{n^{2}\left(a_{n}{ }^{2}+\beta_{n}{ }^{2}\right)} \\
& \frac{\cosh 2 a_{n}(b-r)+\cos 2 \beta_{n}(b-r)}{\cosh 2 a_{n} d-\cos 2 \beta_{n} d} d r \\
& =\mathrm{I} 28 s^{2} p^{2} \pi d a \sigma \sum_{n=\mathrm{I}}^{n=\infty} \frac{\mathrm{I}}{n^{2}\left(a_{n}{ }^{2}+\beta_{n}{ }^{2}\right)} \\
& \frac{\frac{\mathrm{I}}{a_{n}} \sinh 2 a_{n} d+\frac{\mathrm{I}}{\beta_{n}} \sin 2 \beta_{n} d}{\cosh 2 a_{n} d-\cos 2 \beta_{n} d}
\end{aligned}
$$

When $d$ is small ( $\mathrm{r}$ or $2 \mathrm{~mm}$ ), and the frequency is fairly high, then to a very high degree of approximation we can neglect $\sin 2 \beta_{n} d$ and $\cos 2 \beta_{n} d$ as compared with $\sinh 2 a_{n} d$ and $\cosh 2 a_{n} d$, and under these conditions $\sinh 2 a_{n} d$ will be very nearly equal to $\cosh 2 a_{n} d$, and therefore equation (I 4 ) will reduce to

$$
R^{\prime}=\mathrm{I} 28 s^{2} p^{2} \pi a d \sigma \sum_{n=1}^{n=\infty} \frac{\mathrm{I}}{n^{2}\left(a_{n}^{2}+\beta_{n}{ }^{2}\right) a_{n}}
$$


The value of $R^{\prime}$, as given by equation ( 15 ), represents the increase in resistance due to the variation of the electric and magnetic fields within the material of conductor. In addition to this we have of course the ordinary resistance of the wire which it offers to direct current, and if we denote this resistance by $R_{0}$, then the total resistance will be

$$
R=R^{\prime}+R_{0}
$$

The following table gives the values of (per cent change in resistance) for two different coils and three distinct frequencies. Column one gives the experimental value of $\frac{R^{\prime}}{R}$ as determined by Wien, ${ }^{7}$ and the other columns gives the values of $\frac{R^{\prime}}{R}$ as calculated by various formulæ. $R$ is the direct current resistance of coil, and $R^{\prime}$ is the change in the resistance due to frequency.

\section{TABLE.}

Coil I. $\quad \mathrm{r}-0.0485 \mathrm{~cm} . \quad \mathrm{s}=8.75$.

\begin{tabular}{|c|c|c|c|c|c|}
\hline Frequency & $\frac{\mathrm{R}^{\prime}}{\mathrm{R}}$ measured & $\begin{array}{l}R^{\prime} \text { by Wien's } \\
R \text { formula }\end{array}$ & $\begin{array}{l}R^{\prime} \text { by Sommer } \\
R \text { feld's form }\end{array}$ & $\frac{R^{\prime} \text { by Picciati }}{R}$ formula & $\begin{array}{l}\mathrm{R}^{\prime} \text { by formula } \\
\mathrm{R}_{(\mathrm{x})}\end{array}$ \\
\hline $2 \pi \times 4050$ & 0.021 & 0.023 & 0.042 & 0.032 & 0.023 \\
\hline $2 \pi \times 5680$ & 0.045 & 0.045 & 0.080 & 0.062 & 0.044 \\
\hline $2 \pi \times 8310$ & 0.089 & 0.100 & 0.165 & 0.125 & 0.090 \\
\hline
\end{tabular}

Coil II. $\quad \mathrm{r}=0.1 \mathrm{~cm} . \quad \mathrm{s}=4.57$.

\begin{tabular}{l|l|l|l|l|l}
\hline $2 \pi \times 4050$ & 0.34 & 0.521 & 0.58 & 0.45 & 0.365 \\
$2 \pi \times 5680$ & 0.60 & 1.045 & 0.94 & 0.75 & 0.59 \\
$2 \pi \times 8310$ & 0.89 & 2.053 & 1.37 & 1.11 & 0.89 \\
\hline
\end{tabular}

The agreement between the observed values and those calculated by my formula is exceedingly good, which shows that the deduction of the formula on the assumption that the winding is of square section leads to accurate results. The formula ( $\mathrm{I}_{5}$ ) is very convenient 
for calculation, the series is very rapidly converging, in fact for all ordinary cases where the wire is fairly fine and the frequency is anywhere above 2,000, the first term will suffice. An examination of equation (I 5) will show that the change in resistance is a function of the frequency, of the diameter of winding, and also of the number of turns per unit length or the pitch of the winding.

When the frequency is very high, say $10^{6}$, and the diameter of the wire is not exceedingly small, then the value of $R$ as given by equation (I5) will reduce to a still simpler form, for in that case we may neglect $\frac{n^{4} \pi^{4}}{d^{4}}$, as compared with $\mathrm{x} 6 \pi^{2} \sigma^{2} p^{2}$, and the value of $a$ and $\beta$ will be as follows:

$$
a=\beta=\sqrt{2 \pi \sigma p}
$$

Introducing these values of $a$ and $\beta$ into equation (I 5) we get

$$
\begin{aligned}
R^{\prime} & =\frac{\mathrm{I} 28 \mathrm{~s}^{2} p^{2} \pi d a \sigma}{4 n \sigma p \sqrt{2 n \sigma p}} \sum_{n=1}^{n=\infty} \mathrm{I} \\
n^{2} & =\frac{32 s^{2} d a \sqrt{p}}{\sqrt{2 \pi \sigma}} \sum_{n=1}^{n=\infty} \frac{I}{n^{2}}
\end{aligned}
$$

The resistance per unit length is

$$
R^{\prime}=\frac{I 6 s^{2} \sqrt{f} d}{\pi \sqrt{ } \sigma} \sum_{n=1}^{n=\infty} \frac{I}{n^{2}}
$$

The total resistance will be

$$
\begin{aligned}
R= & R^{\prime}+R_{0}=R_{0}+\frac{\mathrm{I} 6 s^{2} \sqrt{f} d}{\pi \sqrt{\sigma}} \sum_{n=1}^{n=\infty} \frac{\mathrm{I}}{n^{2}} \\
& =R_{0}\left\{\mathrm{I}+4 s^{2} \sqrt{f \sigma} d^{3} \sum_{n=1}^{n=\infty} \frac{\mathrm{I}}{n^{2}}\right\}
\end{aligned}
$$

It is interesting to compare the resistance of a solenoid at such high frequency with that of a straight wire of same length. The 
resistance of a straight cylindrical conductor per unit length at a very high frequency is

$$
\begin{gathered}
R=\sqrt{\frac{\mathrm{I}}{2} p R_{0}}=R_{0} \sqrt{\frac{\frac{\mathrm{I}}{2} \sigma d^{2} \pi p}{4}} \\
=R_{0} \frac{\mathrm{I}}{2} \pi d \sqrt{\sigma} f
\end{gathered}
$$

Now, if the frequency is very high, we can neglect unity as compared with the remaining term in (I6), and therefore the ratio of the resistance of coil to that of straight conductor will be

$$
\frac{R_{\mathrm{c}}}{R_{\mathrm{w}}}=\frac{4 s^{2} \sqrt{f \sigma} d^{3}}{\frac{\mathrm{I}}{2} \pi d \sqrt{f \sigma}} \sum_{n=1}^{=\infty} \frac{\mathrm{I}}{n^{2}}=\frac{8 s^{2} d^{2}}{\pi} \sum_{n=1}^{n=\infty} \frac{\mathrm{I}}{n^{2}}
$$

Equation (I8) shows that for high frequencies the ratio $\frac{R_{c}}{R_{\mathrm{w}}}$ is independent of frequency, the ratio increases as the square of diameter of wire and inversely as the square of the pitch. The same conclusions were arrived at by Battelli by an entirely different method, and were verified by Blake experimentally.

\section{EXPERIMENTAL RESULTS.}

Though my theory agrees so closely with the experimental results of Wien, yet it was thought desirable to verify it further by additional experiments. Professor Pupin kindly consented to let me use his high-frequency machine, and the experiments were therefore conducted in his laboratory. The method-adopted in measuring the resistance was the ordinary bridge method. To balance for the inductance I used a torroidal coil, which Professor Pupin had in his laboratory. The coil is wound with stranded wire of very small diameter, the whole diameter of the wire not being more than $0.2 \mathrm{~cm}$, and this is made up of 30 wires, so that we can safely neglect the change in resistance in the balancing coil. As a detecting instrument I used a tuned telephone which Professor Pupin employs in some of his investigations. It is probably 50 times as sensitive as the ordinary telephone receiver. The method of procedure was to 
determine the resistance of the coil by direct current, and then to measure its resistance in the bridge for alternating current. Two solenoids wound with wires of different diameters were employed. The frequencies were maintained constant by the aid of a tuning fork.

The dimensions of the coil are as follows:

Coil I:

Internal radius of coil diameter of wire $=4.96 \mathrm{I} \mathrm{cm}$ Number of turns per $\mathrm{cm}$ total number of turns resistance of coil

$=0.29 \mathrm{~cm}$

$=3.1433$

$=476$

Coil II :

$=0.44 \mathrm{I}$ ohms

Internal diameter of coil

$=4.96 \mathrm{r} \mathrm{cm}$

diameter of wire

$=0.206 \mathrm{~cm}$

Number of turns per cm

$=4.319$

total number of turns

$=580$

resistance of coil

$=1.028 \mathrm{ohms}$

The results obtained are given in the following table:

Coil I.

\begin{tabular}{c|c|c}
\hline Frequency & $\frac{R^{\prime}}{\mathrm{R}}$ measured & $\frac{\mathrm{R}^{\prime}}{\mathrm{R}}$ calculated \\
\hline $2 \pi \times 1280$ & 0.0159 & 0.160 \\
$2 \pi \times 896$ & 0.091 & 0.084 \\
\hline
\end{tabular}

Coil II.

\begin{tabular}{l|l|l}
\hline $2 \pi \times 1280$ & 0.052 & 0.044 \\
$2 \pi \times 896$ & 0.027 & 0.022 \\
\hline
\end{tabular}

The experimental values of the resistances could not be relied upon within .005 ohms, and hence the values may be modified slightly, which may either improve the agreement between the calculated and measured values or make it worse. The small difference between the measured and calculated values may be accounted for 
if we bear in mind that, to get the final expression for the change in resistance, we have replaced the Bessel functions by exponential functions which is more nearly true as the argument gets large. Now, since the argument is a function of the frequency and of the radius of coil it is evident that for higher frequencies the approximation will be closer than for lower frequencies and this may account for the small discrepancy at the lower frequency. By final formula (I 5) therefore as it stands will hold absolutely for all cases where the argument (kr) is fairly large, say, 20 or more, which implies a fairly high frequency, perhaps I,500 or more, and the radius of the coil must not be very small. We can of course vary the two factors, making one very large and one very small, but so long as the product $k r$ is not small the formula will hold. If we have a low frequency and the radius of the coil is also small, then the final expression (I 5) will not be strictly true, and it will approximate the true value as $\mathrm{kr}$ gets larger.

\section{CHANGE IN INDUCTANCE.}

The change of inductance can be obtained by a method similar to that employed in obtaining the change in resistance. In this case we make use of the equality of electro-magnetic energy and electro-kinetic energy,

or

$$
\begin{gathered}
\frac{I}{8 \pi} \int H^{2} d v=\frac{I}{2} \mathcal{L} I^{2} \\
\mathcal{L}=\frac{\mathrm{I}}{4 \pi I} \int H^{2} d v
\end{gathered}
$$

Now

$$
H^{2}=L^{2}+M^{2}
$$

The values of $L$ and $M$ are given by equations (7)

$$
\begin{aligned}
& \left.L=\sum_{n=1}^{n=\infty} \frac{m_{n}}{i p} \mid A^{\prime} J_{1}\left(i k_{n} r\right)+B^{\prime}{ }_{n} Y_{1}\left(i k_{n} r\right)\right\} \frac{4}{n \pi}(i)^{n-1} \sin \frac{n \pi z}{d} e^{i p i} \\
& M=\sum_{n=1}^{n=\infty} \frac{k_{n}}{p}\left\{A^{\prime}{ }_{n} J_{0}\left(i k_{n} r\right)+B^{\prime}{ }_{n} Y_{0}\left(i k_{n} r\right)\right\}{ }_{n \pi}^{4}(i)^{n-1} \cos \frac{n \pi z}{d} e^{i p t}
\end{aligned}
$$


Introducing the values of the constants $A^{\prime}$ and $B^{\prime}$ from equations (xI) and using the approximate values of the Bessel's functions we get:

$$
\begin{aligned}
& L=\sum_{n=1}^{n=\infty} \frac{\mathrm{I} 6 m_{n} s I}{k_{n} n}(i)^{n-1}\left\{\frac{e^{k_{n}(b-1)}+e^{-k_{n}(b-r)}}{e^{-k_{n} d}-e^{k_{n} d}}\right\} \frac{\sqrt{ } a}{\sqrt{r}} \sin \frac{n \pi z}{d} \\
& M=\sum_{n=1}^{n=\infty} \frac{16 m_{n} s I}{n}(i)^{n-1}\left\{\frac{e^{k_{n}(b-r)}-e^{-k_{n}(b-r)}}{e^{-k_{n} d}-e^{k_{n} d}}\right\} \frac{\sqrt{ } a}{\sqrt{r}} \cos \frac{n \pi z}{d}
\end{aligned}
$$

Now

$$
H^{2}=L^{2}+M^{2}
$$

and since $H, L$, and $M$ are complex quantities, the square of the amplitude can be obtained by multiplying the quantity by its conjugate, which in this case will be merely putting $(-i)$ everywhere in place of $(i)$, and this will give us

$$
\begin{aligned}
& H^{2}=\sum_{n=1}^{n=\infty} \frac{256 s^{2} I^{2}}{n^{2}\left(a_{n}{ }^{2}+\beta_{n}^{2}\right)} \\
& \left\{\frac{e^{2 a_{n}(b-r)}-e^{-2 a_{n}(b-r)}+e^{2 i \beta_{n}(b-r)}+e^{-2 i \beta_{n}(b-r)}}{e^{2 a_{n} d}+e^{-2 a_{n} d}-e^{-2 i \beta_{n} d}-e^{2 i \beta_{n} d}}\right\} \frac{a}{r} \sin ^{2} \frac{n \pi z}{d} \\
& +\sum_{n=1}^{n=\infty} \frac{256 s^{2} I^{2}}{n^{2}}\left\{\frac{\left.e^{2 a_{n}(b-r)}-e^{-2 a_{n}(b-r)}-e^{-2 i \beta_{n}(b-r}\right)-e^{2 i \beta_{n}(b-r)}}{e^{2 a_{n} d}-e^{-2 a_{n} d}-e^{-2 i \beta_{n} d}-e^{2 i \beta_{n} d}}\right\} a \cos ^{2} \frac{n \pi z}{d} \\
& =\sum_{n=1}^{n=\infty} \frac{256 s^{2} I^{2}}{n^{2}\left(a_{n}{ }^{2}+\beta_{n}{ }^{2}\right.}\left\{\frac{\cosh 2 a_{n}(b-r)-\cos 2 \beta_{n}(b-r)}{\cosh 2 a_{n} d-\cos 2 \beta_{n} d}\right\} \frac{\alpha}{r} \sin \frac{n \pi z}{d} \\
& +\sum_{n=1}^{n=\infty} \frac{1256 s^{2} I^{2}}{n^{2}}\left\{\frac{\cosh 2 a_{n}(b-r)-\cos 2 \beta_{n}(b-r)}{\cosh 2 a_{n} d-\cos 2 \beta_{n} d} \mid \frac{\alpha}{r} \cos ^{2} \frac{n \pi z}{d}\right.
\end{aligned}
$$

For one single turn,

$$
\int H^{2} d z^{\prime}=\int_{0}^{* \pi} \int_{a}^{b} \int_{-\frac{d}{2}}^{+\frac{d}{2}} H^{2} r d r d z d \theta=2 \pi \int_{a}^{b} \int_{-\frac{d}{2}}^{+{ }^{d}} H^{2} r d r d z
$$


and

$$
\mathcal{L}=\frac{\mathrm{I}}{4 \pi I^{2}} \int H^{2} d v=\frac{\mathrm{I}}{2 I^{2}} \int_{a}^{b} \int_{-\frac{d}{2}}^{+\frac{d}{2}} H^{2} r d r d z
$$

Introducing value of $H^{2}$ from equation (2I) we get:

$$
\begin{gathered}
\mathcal{L}=\mathrm{I} 28 s^{2} \alpha\left[\sum _ { n = 1 } ^ { n = \infty } \int _ { a } ^ { b } \int _ { - \frac { d } { 2 } } ^ { + \frac { d } { 2 } } \left\{\frac{\mathrm{I}}{a_{n}{ }^{2}+\beta_{n}{ }^{2}} \frac{\cosh 2 a_{n}(b-r)-\cos 2 \beta_{n}(b-r)}{\cosh 2 a_{n} d-\cos 2 \beta_{n} d}\right.\right. \\
\sin ^{\frac{{ }^{n} 2 \pi z}{d}} \\
\left.\left.+\frac{\cosh 2 a_{n}(b-r)-\cos 2 \beta_{n}(b-r)}{\cosh 2 a_{n} d-\cos 2 \beta_{n} d} \cos \frac{{ }^{2} n \pi z}{d}\right\} d r d z\right] \\
=\mathrm{I} 28 s^{2} a d \sum_{n=1}^{n=\infty} \frac{\mathrm{I}}{n^{2}}\left(\frac{\mathrm{I}}{a_{n}{ }^{2}+\beta_{n}{ }^{2}}+\mathrm{I}\right) \frac{\frac{1}{2 a_{n}} \sinh 2 a_{n} d-\frac{1}{2 \beta_{n}} \sin 2 \beta_{n} d}{\cosh 2 a_{n} d-\cos 2 \beta_{n} d}
\end{gathered}
$$

When the frequency is very high and the diameter of wire is not very large, $a$ will be a large quantity and equation (22) will reduce to the following:

$$
\mathcal{L}=64 s^{2} \alpha d \sum_{n=1}^{n=\infty}\left(\frac{\mathrm{I}}{a_{n}{ }^{2}+\beta_{n}{ }^{2}}+\mathrm{I}\right) \frac{\mathrm{I}}{a_{n}{ }^{2} n^{2}}
$$

This is the inductance due to the magnetic field within the material of winding of a single turn, and the inductance due to the total number of turns will be

$$
\mathcal{L}=64 s^{3} d a l \sum_{n=1}^{n=\infty} \frac{\mathrm{I}}{a_{n} n^{2}}\left(\frac{\mathrm{I}}{a_{n}^{2}+\beta_{n}^{2}}+\mathrm{I}\right)
$$

Where $l$ is the length of the solenoid. The change in inductance will be the difference between the inductance, due to magnetic field within the material of the coil, when the current is continuous and alternating. For continuous current the inductance due to magnetic field in the material of the winding alone is ${ }^{8}$

$$
\mathcal{L}=\frac{\mathrm{I}}{6} 4 \pi^{2} s^{2} l d(4 a+d) .
$$


Hence the change in inductance $\mathcal{L}^{\prime}$ will be

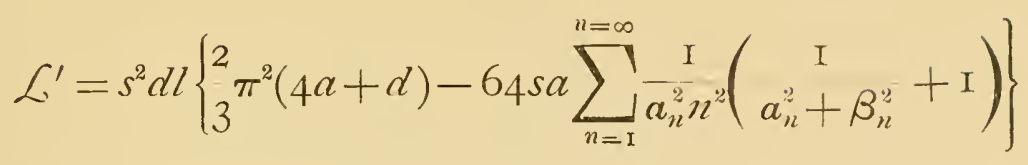

The per cent change in inductance will be the ratio of change in inductance to the total inductance of solenoid. Now the total inductance of solenoid consists of two parts; one due to magnetic field within the solenoid, and the other due to magnetic field within material of the windings, thus:

$$
\mathcal{L}=4 \pi^{2} s^{2} l a^{2}+\frac{\mathrm{I}}{6} 4 \pi^{2} s^{2} l d(4 a+d) .
$$

If $a$ is not very small, then we may put

$$
\mathcal{L}=4 \pi^{2} s^{2} l a^{2}
$$

If we denote the per cent change in inductance by $\mathcal{L}_{2}$, then we have

$$
\begin{gathered}
\mathcal{L}_{2}=\frac{s^{2} d l\left\{\frac{2}{3} \pi^{2}(4 a+d)-64 s a \sum_{n=1}^{n=\infty}\left(\frac{\mathrm{I}}{a_{n}^{2}+\beta_{n}^{2}}+\mathrm{I}\right)\right\}}{4 \pi^{2} s^{2} l^{2}} \\
=\frac{d\left\{\frac{2}{3} \pi^{2}-\mathrm{I} 6 s \sum_{n=1}^{n=\infty} \frac{\mathrm{I}}{a_{n}^{2} n^{2}}\left(\frac{\mathrm{I}}{a_{n}^{2}+\beta_{n}^{2}}+\mathrm{I}\right)\right.}{\pi^{2} a} .
\end{gathered}
$$

As an example, suppose $a=5 \mathrm{~cm}, d=0.2 \mathrm{~cm}, s=4$

and

and

$$
p=2 \pi \times \mathrm{IO}^{6} a^{2}=\beta^{2}=23.6 \times \mathrm{IO}^{3}, a=\mathrm{I} 54
$$

$\mathcal{L}_{2}=\frac{0.2\left\{\frac{2}{3} \times \mathrm{IO}-\mathrm{I} 6 \times 4 \frac{\mathrm{I}}{\mathrm{I} 54}\left(\mathrm{I}+\frac{\mathrm{I}}{9}+\ldots\right)\right.}{50}=0.025$ approximately.

The change in inductance for this particular case is about 2.5 per cent. 
Résumé.-To sum up the results of this investigation we can say that the theory developed in this paper for the change in resistance and inductance of solenoids due to frequency is fully corroborated by experimental facts. The final expressions given by equations (15) and (26) are very simple and convenient for calculation, and will give accurate results in all cases except when the frequency is exceedingly low. For convenience of reference I reproduce here equations (I5) and (26), indicating also the meaning of the factors involved.

$$
\begin{aligned}
& R^{\prime}=\mathrm{I} 28 s^{2} p^{2} \pi d a \sigma \sum_{n=1}^{n=\infty} \frac{\mathrm{I}}{n^{2}\left(a_{n}^{2}+\beta_{n}^{2}\right) a_{n}} \\
& \mathcal{L}^{\prime}=s^{2} d l\left\{\begin{array}{l}
2 \\
\pi^{2}(4 a+d)-64 s a \sum_{n=1}^{n=\infty} a_{n}^{2} n^{2}
\end{array}\left(\frac{\mathrm{I}}{a_{n}^{2}+\beta_{n}^{2}}+\mathrm{I}\right)\right\}
\end{aligned}
$$

$R^{\prime}$ is the change in resistance due to frequency.

$\mathcal{L}^{\prime}$ " " " "inductance " "

$$
\begin{gathered}
a^{2}=\frac{m^{2}+\sqrt{m^{4}+\mathrm{I} 6} \pi^{2} \sigma^{2} p^{2}}{2} \\
\beta^{2}=\frac{-m^{2}+\frac{\sqrt{m^{4}+\mathrm{I} 6 \pi^{2} \sigma^{2} p^{2}}}{2}}{m=\frac{n \pi}{d}(n=\mathrm{I}, 3,5,7,9 \ldots \ldots)}
\end{gathered}
$$

$d$ denotes diameter of wire.

$\sigma$ " conductivity.

$p$ " " $2 \pi$ frequency.

$s \quad$ " number of turins per unit length.

a " internal radius of solenoid.

$l$ " length of solenoid.

In conclusion, I wish to express my thanks to Prof. M. I. Pupin, of the Columbia University, for his kindness in permitting me to use his private laboratory, where the experimental part of the work was carried out, and I also wish to thank his assistant, Mr. W. E. Cushman, for his kind assistance in the experimental work.

Washington, August I6, I907. 